\title{
Memory for forms: Common memory formats for verbal and visual stimulus presentations
}

\author{
GREGORY L. MURPHY and J. WESLEY HUTCHINSON \\ Stanford University, Stanford, California 94305
}

\begin{abstract}
Numerous experiments have attempted to show that people use a visuospatial representation to remember pictures and objects by demonstrating that perceptual variables affect memory performance. One problem with such experiments is that perceptual differences in the stimuli could cause differences in a verbal representation as well (i.e., could lead to different descriptions). The three experiments reported here use as stimuli a set of geometric forms designed to have identical verbal descriptions, yet to differ in visual complexity. Results showed that recall differences could be predicted on the basis of visual complexity whether the stimuli were described or presented visually. Furthermore, this result cannot be attributed to a response bias in reproducing the stimuli. Various models of memory for forms are considered in the light of these results, as well as the "tacit knowledge" explanation for mental imagery phenomena.
\end{abstract}

The purpose of this paper is to investigate the relationship between verbal and visual modes of acquiring information about physical objects and to demonstrate the use of a visual representation in memory even when information is presented verbally. Specifically, we present a method in which geometric figures are described so that the description's verbal information is held constant, but the figures vary in visual complexity. In this way, the effects of visual coding of the stimuli may be demonstrated without the potential confound of differences in verbal memory.

Recent experimental studies have provided strong evidence that there is a specifically visual form of mental representation that can be activated in certain contexts (e.g., Cooper \& Shepard, 1973; Finke, 1979; Kosslyn, 1975; Kosslyn, Ball, \& Reiser, 1978; Podgorny $\&$ Shepard, 1978). Commonalities between this form of representation and perceptual representations are often hypothesized in order to explain behavior in mental imagery tasks (Finke, 1980; Shepard, 1981). (Although we call these representations "visual," it is likely that they are "visuospatial" in character. Our experiments do not separate visual and spatial information; we use the term "visual" only to ease exposition.)

Although such explanations seem to be gaining wide acceptance, a more controversial question is whether

This research was supported by NSF Grant BNS- $80-05517$ awarded to Roger Shepard. The authors gratefully acknowledge the comments of Ronald Finke, Brian Ross, and Roger Shepard on earlier versions of this paper. Please address reprint requests to Gregory Murphy, Department of Psychology, Building 420Jord an Hall, Stanford University, Stanford, California 94305, or to Wes Hutchinson, Matherly Hall, Marketing Department, College of Business Administration, University of Florida, Gainesville, Florida 32611. visual representations can be used to explain performance in memory tasks (rather than in judgment and transformation tasks, as in the studies cited above). In spite of early successes in attempts to do so (Bower, 1970b; Paivio, 1969, 1971), powerful alternative explanations were soon proposed (see Bower, 1970a; Bower, Munoz, \& Arnold, cited in Anderson \& Bower, 1973).

More recently, studies of mental maps have suggested that mental imagery has an important function in representing location and orientation information in memory (Neisser, 1976). Although many researchers in this area prefer to separate their investigations from the question of representational format, there are some who have contrasted visual and verbal codes in map memory (Foos, 1980; Stevens \& Coupe, 1978).

One strategy for attempting to demonstrate that there is a separate visual format in memory has been to vary encoding instructions given to subjects. For example, when given imagery mnemonic instructions, subjects recall paired associates far better than controls (see Bower, 1970b, for a review). Another strategy has been to specify either categorical or physical properties of stimuli as a basis for making decisions (e.g., Kosslyn, 1975; Posner, Boies, Eichelman,\& Taylor, 1969; Tversky, $1969,1974)$. However, these strategies seem open to the criticism that different processes (rather than different types of representation) can account for reaction time and recall effects. Also, there have been many studies comparing picture and word memory, but any differences between the two might be attributed to different information available in each type of stimulus.

The evidence needed to demonstrate a visual form of memory representation is that provided by experiments in which visual aspects of the stimuli are varied but in which the verbal aspects are held constant over stimuli. 
If people do not construct visual representations of such stimuli, then memory performance should be the same for all of them, or, at least, unrelated to variations in visual quality and form. If people can and do form visual representations, stimuli with the most coherent perceptual structure should be easiest to remember. Of course, the theoretical problem is not to show that verbal information is ignored, or that there is no verbal or amodal form of representation in memory, but rather to give a convincing example of when these representations cannot account for memory differences and when a visual representation can.

In our experiments, we attempted to control for the nonvisual factors by using as stimuli unfamiliar polygons (see Figure 1) that varied greatly in perceived complexity, but that are all permutations of the same elements on a 4 by 4 grid. Each stimulus was described by telling the subjects what element to remember in each square of the grid, so that the verbal content was the same for all stimulus descriptions and only the arrangement of the elements was varied. As we argue later, it is implausible that orderly effects should arise through verbal factors in this experiment, whereas the results are clearly interpretable on the basis of visual complexity.

All three experiments used the same stimuli, so we shall give some attention to their construction. Since there is a large number of possible combinations of the basic elements we used, we restricted the stimuli to those that varied systematically along these dimensions: symmetry-asymmetry, number of figures (one or two), number of continuous edges (ranging from 10 to 20),

A

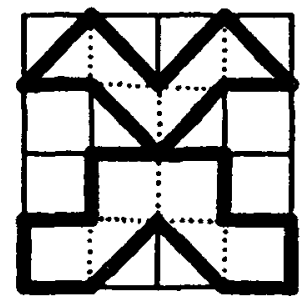

$\mathrm{B}$

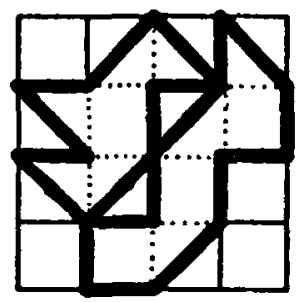

C
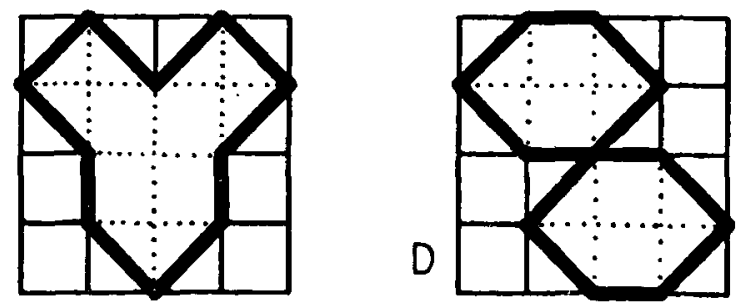

Figure 1. Examples of stimuli used in the three experiments. All are composed of the same 16 elements. The two in the left column are symmetric; the two in the top row have six parts, and those in the bottom row have four parts. The number of edges are $19,20,12$, and 10 for $A, B, C$, and $D$, respectively. and number of "parts" (four or six). The number of parts was measured by the minimum number of triangles and/or quadrilaterals necessary to construct the figure and was motivated by a notion that subjects may be "chunking" the figures into groups of triangles and rectangles.

Our main purpose in this stimulus design was to sample a wide range of visual complexity in the stimuli, and the results indeed showed a range of difficulty in these figures. Another reason was to provide some information as to which of these dimensions are important in encoding and remembering the stimuli.

The final main question of interest is the comparison of visual and verbal forms of stimulus presentation. We believe that performance is based on the same type of memory representation in both cases, so that the patterns of results should be very similar for the two. However, there are certain important differences between the two modes that may result in recall differences. One difference is the accessibility of global features. In general, a short glance at a figure will reveal its shape and orientation. On the other hand, our descriptions use only small elements, so global characteristics must be inferred from remembered elements. Thus, memory for visually presented stimuli may use global perceptual information that is difficult to compute when hearing discrete descriptions. This hypothesis is tested in Experiment 2 .

\section{EXPERIMENT 1}

In the first experiment, subjects heard figures described by a verbal code and then recalled them by drawing them. This experiment attempted to demonstrate that visual complexity affects memory for verbally communicated material, even when the verbal content is controlled. (By "verbal content," we mean the identity of the elements in the memory set.)

\section{Method}

Subjects. Thirty-two Stanford undergraduates participated in the experiment to fulfill a course requirement.

Stimuli. The 16 polygons described above were used as stimuli, with 2 additional practice figures. All the figures were composed of the same elements of a 4 by 4 grid: There were four "blank" (white) cells, four "solid" (black) cells, two cells each with the upper left half, lower left half, upper right half, and lower right half solid (described as "upper left," "lower left," etc.).

The figures varied on these factors: symmetry/asymmetry, number of figures (one or two), and number of parts (six or four). Symmetry was defined as mirror image identity across either the vertical or horizontal axis. A stimulus was considered to have one figure if all its solid areas were connected by an adjacent edge. Stimuli with two figures contained two such connected areas. The number of parts was the number of triangles or quadrilaterals of any size required to represent the stimulus. Since there are many combinations of triangles and quadrilaterals in each stimulus, we used the smallest number that was necessary to construct the stimulus as our "parts" measure. (For example, in Figure 1, Stimulus $\mathrm{C}$ has four parts: 
two triangles and two parallelograms; Stimulus $A$ has six parts: three triangles, one rectangles, and two trapezoids. It is possible to divide each into more, but not into fewer parts.) Within each combination of the factors are two stimuli, which vary as to how many edges they have. Because it is impossible to create a figure with many parts but few edges (or vice versa), the data analysis combines the parts-and-edges factor into one factor, which we call "figure complexity," with four levels: (1) four parts, fewer than 14 edges, (2) four parts, 14 edges, (3) six parts, 14 edges, (4) six parts, more than 14 edges. (Obviously, we do not claim that perceived complexity depends only on the number of edges and parts in the figures, although these are certainly strong factors; see Hochberg \& Brooks, 1960, for a full discussion of perceptual complexity.) Copies of all the stimuli with the number of parts and edges of each are available from the authors upon request.

Because there may be differences in which "view" of a figure is easiest to encode, the figures were described from two viewpoints, one a 90 deg rotation of the other. Two groups of subjects heard one description and two groups heard the description of the rotated figures.

Procedure. Subjects were shown a sample figure (not used in the experiment) to illustrate the code for describing the pictures. An experimenter gave the description and pointed to each cell as he described it. All the subjects readily understood the code and the task. After the experimenter gave the instructions, two practice trials with feedback were given, and subjects were encouraged to ask questions about the task.

While listening to the descriptions, subjects were told to study the grid in front of them and to imagine the appropriate element in each cell as they heard it. The experimenter gave the codes for the cells in a left-to-right and top-to-bottom order (row by row) for two groups and in a top-to-bottom and leftto-right order (column by column) for two groups. The stimulus presentation was timed with a stopwatch so that the codes were read once every $2.5 \mathrm{sec}$. After the description was finished, there was a $10-\mathrm{sec}$ delay before subjects were given the signal to recall (by drawing on the grid) the figure just described. After subjects finished recalling the figure, they were shown the correct one.

Subjects were run in four groups of from 6 to 10 people, and the figures were presented to two groups in one random order and to the other two in another random order.

\section{Results}

As expected, the visual qualities of the stimuli had a marked effect on recall performance. Errors were counted on a cell-by-cell basis, so there was a maximum of 16 possible errors per stimulus. The data were submitted to an analysis of variance with subjects, number of figures, symmetry, and figure complexity as factors. The main effects of symmetry and figure complexity were significant $[\mathrm{F}(1,31)=18.94, \mathrm{MSe}=3.68, \mathrm{p}<.001$, and $F(3,93)=29.01, \mathrm{MSe}=8.27, \mathrm{p}<.001$, respectively]. Overall, symmetric figures were recalled with 2.4 errors/stimulus, and asymmetric figures induced 3.2 errors. Errors increased with increasing levels of figure complexity: $1.2,2.4,3.2$, and 4.5 errors/stimulus for Levels 1-4, respectively. The difference between the second and third levels is relatively small, which may indicate that there is no real effect of parts, but only of number of edges (since Levels 2 and 3 differ only in number of parts). We tested the effect of parts by a contrast between the second and third levels and found that the .8 difference was reliable $[F(1,93)=4.91$, $\mathrm{p}<.05]$.
Table 1

Errors per Stimulus for Experiment 1: Verbal Descriptions

\begin{tabular}{cccccc}
\hline & \multicolumn{4}{c}{ Number of Edges } \\
\cline { 2 - 3 } & \multicolumn{2}{c}{ Four Parts } & & \multicolumn{2}{c}{ Six Parts } \\
\cline { 2 - 3 } \cline { 5 - 6 } & Under 14 & 14 & & 14 & Over 14 \\
\hline One Figure & & & & \\
Asymmetric & 1.8 & 1.6 & 3.9 & 5.0 \\
Symmetric & 1.0 & 3.2 & 2.3 & 2.8 \\
Two Figures & & & & \\
Asymmetric & .5 & 2.7 & 3.4 & 6.5 \\
Symmetric & 1.5 & 2.1 & 3.1 & 3.6 \\
Mean & 1.20 & 2.38 & 3.17 & 4.46 \\
\hline
\end{tabular}

There were no differences in performance for onefigure and two-figure stimuli, inducing 2.7 and 2.9 errors/ stimulus, respectively $(\mathrm{F}<1, \mathrm{MSe}=7.52)$. The interaction of number of figures and symmetry was minimal, but there was a marginally significant interaction of number of figures and figure complexity $[F(3,93)=2.69$, $\mathrm{MSe}=5.36, \mathrm{p}<.06]$. Furthermore, there was a very reliable interaction of symmetry and complexity $[F(3,93)=13.80, \mathrm{MSe}=4.25, \mathrm{p}<.001]$, which seemed to indicate that the symmetry effect holds only for the more difficult figures (the two highest levels of figure complexity). All of these interactions are mitigated by the significant second-order interaction of figure complexity, number of figures, and symmetry $[F(3,93)=$ $5.04, \mathrm{MSe}=5.23, \mathrm{p}<.005]$, as shown in Table 1 . Since there is only one stimulus in each cell of the interaction, it is confounded with any idiosyncracies of individual stimuli. Perhaps its most straightforward interpretation is that there are differences between the stimuli in addition to the factors we used to generate them.

As Table 1 shows, there were striking differences among the stimuli, with error rates ranging from .5 to 6.5 errors/figure. The most potent factor appears to be figure complexity, although it is most effective for asymmetric figures. The information value of symmetry seems to lower error rates overall, but its effect can only be manifested on the more difficult stimuli (because of the floor effect for easier ones), leading to the interaction.

\section{Discussion}

As expected, the figures most difficult to recall were asymmetric ones with the highest level of complexity; the easiest figures were the least complex. Perhaps not surprisingly, number of figures had no main effect once numbers of edges and parts were controlled for. It is clear that subjects must have been "chunking" the elements into larger parts (or else they would not perform so well on the easiest stimuli); perhaps the lack of an effect of the number of figures indicates that the figures are too large to use as chunks. In other words, it is not easier to remember two simple figures than one complex 
one, if each stimulus can be decomposed into the same number of chunks.

Since all stimuli are composed of the same elements, the results clearly indicate that subjects are not simply storing the elements in association chains, since this would produce no differences among the stimuli. The error rates are predicted by minimal assumptions of difficulty in perceptual memory (i.e., the effects and interaction of symmetry and complexity discussed above), which supports the claim that subjects use a picture-like representation.

One possible objection to our conclusion is that subjects develop verbal grouping strategies to organize the figures as they hear the description. It may just be that these strategies work best for the simpler figures, and so the results only appear to implicate use of visual representations. Although this sort of argument can never be fully disconfirmed (since it is quite vague), one way of rendering it implausible is to show that the results of this experiment are quite close to those of an analogous visual memory experiment. If subjects who see the whole figures at once give the same results as subjects who hear descriptions, the most parsimonious explanation will be an underlying representation common to the two tasks. (Of course, this representation need not necessarily be pictorial, an issue we raise in the general discussion.)

\section{EXPERIMENT 2}

Experiment 2 is a visual memory analogue of the first experiment. Here, subjects were shown slides of the stimuli for a brief time and, after a 15 -sec delay, recalled them as before. This experiment allows us to test for some predicted differences between the verbal description and visual presentation modes. The main difference expected was in the effect of symmetry. Since the verbal description is inherently piecemeal, sometimes subjects may not have noticed symmetry, especially when the axis of symmetry was parallel to that of the description order (since in that case the symmetry does not appear until more than half the stimulus has been described--i.e., until the line of symmetry has been crossed). Thus, we expect the effect of symmetry to be somewhat stronger with visual presentation.

Another difference might be in the type of errors people make in the two kinds of stimulus presentation. Since it is easier to observe gestalt properties of the stimulus (overall shape), in visual presentation, we expected more errors that would preserve local but not global configuration in the description group. While scoring the responses of the first experiment, we noticed that subjects sometimes reproduced a correct portion of a figure in the wrong orientation. Such errors create gross distortions of the shape and, so, would not be expected in visual presentation. These errors were systematically scored, and the performances of the two presentation conditions were compared.

\section{Method}

Subjects. Subjects were 28 Stanford undergraduates who participated to fulfill a course requirement.

Stimuli. The same stimuli used earlier were photographed onto slides. The solid areas of the figures were colored a light purple, and the lines of the grid could be seen even in the solid areas.

Procedure. Subjects received instructions and practice items as before, except that imagery was not mentioned. Subjects were told to study the picture for as long as it was presented and, after the experimenter signaled, to reproduce it on the grid in front of them.

Each figure was presented for $4 \mathrm{sec}$, followed by a $15-\mathrm{sec}$ delay. The stimuli were projected onto a screen at the front of the room, and subjects were seated at a table from about 4 to $10 \mathrm{ft}$ away from the screen. The figures filled the screen, and no subject reported difficulty seeing them.

Two random orders of stimulus presentation were used, two groups per order. The stimuli were presented in two "views," as before--one view a 90 -deg rotation of the other-counterbalanced with the order of presentation. Thus, there were four counterbalancing groups, with from six to nine subjects in each group.

\section{Results}

The recall data were scored on a cell-by-cell basis, as before, and the average error rate was 3.9 errors/stimulus, somewhat higher than the 2.8 average of Experiment 1 . This difference is not easily interpretable, due to the difference in presentation times of the two experiments (equalizing presentation times in different modalities is generally problematic, given the different sensory and cognitive mechanisms involved). Besides this overall difference, however, the results of the two experiments were remarkably similar. Symmetric stimuli were easier than asymmetric ones: 3.2 vs. 4.6 errors $[F(1,27)$ $=26.80, \mathrm{MSe}=7.49, \mathrm{p}<.001]$. The effect of figure complexity was again very reliable: $1.5,3.4,4.6$, and 6.2 errors for Levels $1-4$ of this factor $[F(3,81)=55.11$, $\mathrm{MSe}=7.78, \mathrm{p}<.001]$. Finally, there was no discernible effect of number of figures; mean errors were 4.0 and 3.9 for one- and two-figure stimuli $(\mathrm{F}<1, \mathrm{MSe}=7.65)$.

The interactions of symmetry and edge-parts and of figures and edge-parts were also significant, but these were again mitigated by the second-order interaction $[F(3,81)=3.82, \mathrm{MSe}=8.69, \mathrm{p}<.025]$, shown in Table 2. Thus, the stimuli show idiosyncracies not

Table 2

Errors per Stimulus for Experiment 2: Visual Presentation

\begin{tabular}{cccccc}
\hline & \multicolumn{4}{c}{ Number of Edges } \\
\cline { 3 - 6 } & \multicolumn{2}{c}{ Four Parts } & & \multicolumn{2}{c}{ Six Parts } \\
\cline { 3 - 6 } & Under 14 & 14 & & 14 & Over 14 \\
\hline One Figure & & & & \\
$\quad$ Asymmetric & 2.4 & 3.1 & 5.5 & 8.0 \\
Symmetric & 1.0 & 4.9 & 3.6 & 3.3 \\
Two Figures & & & & \\
Asymmetric & .8 & 2.3 & 6.5 & 8.2 \\
Symmetric & 2.0 & 3.4 & 2.5 & 5.2 \\
Mean & 1.54 & 3.43 & 4.56 & 6.19 \\
\hline
\end{tabular}


fully accounted for by our factors, just as in the first experiment. Although there are some reversals, the general pattern is one in which difficulty increases with number of edge-parts and in which symmetric stimuli are easier than asymmetic stimuli. The overall difference in numbers may obscure the similarity between these results and those of Experiment 1 (see Table 1). The correlation between the number of errors on the two tasks taken over all items was $.92[\mathrm{t}(14)=8.72$, $\mathrm{p}<.001]$.

One difference between these results and those found in Experiment 1 is that the effect of symmetry was larger for visual presentation (a 1.3error/figure advantage for symmetric stimuli) than for the described stimuli (a .8-error/figure difference) $[t(58)=1.99$, $\mathrm{p}=.05]$. However, this difference may be due to the overall difference in the number of errors in the two tasks (i.e., the difference could be one of scale of measurement), which is a problem of interpretation for all such interactions.

In addition to the simple error analysis, the response forms were scored for triangle inversions. These were cases in which a figure contained a vertex formed by two adjacent diagonal elements (e.g., upper right next to upper left, or lower left above upper left), and the subject's response changed both elements, inverting the vertex to point in the opposite direction. (Also scored as inversions were cases in which subjects made figureground reversals in addition to the inversion, since this also preserved the identity of the part.) Such errors greatly changed the overall shape of the figure, but they presumably indicated knowledge of what part belonged in that location; that is, they demonstrated the use of chunks. The scorer was blind to the expected results and was motivated to find as many such errors as possible.

For the visual presentation group, each subject made a total of from 0 to 6 such inversions, with mean 1.54 and median 1.50 inversions/subject. For the description group (the first experiment), subjects made between 0 and 12 inversions, with mean 2.88 and median 2.0. Thus, there were more inversions for the verbal presentation ( $\mathrm{p}<.01$ by a Mann-Whitney test), even though this group had fewer errors overall.

\section{Discussion}

The close correspondence between the error patterns in the first two experiments suggests that subjects formed equivalent representations in the two tasks. Although it does seem implausible that subjects used a verbal grouping strategy that fortuitously caused effects of figure complexity and symmetry that were almost identical in the visual memory and description conditions, there is still a possible alternative explanation that would predict similar results in the two experiments.

This alternative hypothesis explains the seeming effect of visual complexity in terms of response biases.
In its simplest form, it says that subjects memorize the description verbally as well as they can. Following classic principles of verbal memory (e.g., Atkinson \& Shiffrin, 1968; Glanzer \& Cunitz, 1966), part of the first row can be stored in long-term memory, and the last row's description can be rehearsed in short-term memory until recalled. Once the remembered sections have been recalled on paper, the subject attempts to fill in the rest by making a plausible figure. Obviously, stimuli that appear symmetric would be easier to guess correctly, thus explaining that effect. Since subjects may have a bias to create simpler figures (see Riley, 1962), they will guess incorrectly on the stimuli with greater figure complexity. If this is true, then eliminating the response component of the task should eliminate the bias and result in no differences among the stimuli. The next experiment attempts to remove any possible response bias.

The expected differences between the two presentation modes were also found: Symmetry had a greater effect for visual presentation, and more triangle inversions were found for verbal descriptions in spite of the lower overall error rate. Both effects are related to the ability to extract global properties of the stimuli when presented visually. It is probably difficult to keep a representation of the whole figure activated while adding on a new element every $4 \mathrm{sec}$, thus decreasing the chance of subjects' noticing global properties in that condition.

\section{EXPERIMENT 3}

In the first experiment, subjects were told to imagine the figure on the grid as it was described, but they were not given standard instructions of mental imagery experiments (Kosslyn, 1975; Kosslyn et al., 1978). In this experiment, subjects were instructed to form a complete, clear visual image of the figure as it was described. A further difference was that the rate of presentation of the description was controlled by the subject. He or she gave a verbal signal when ready to hear the element for the next cell. The major dependent measure was therefore the amount of time the subject took to hear the description. We expected that subjects would require more time to construct and rehearse the images of the more complex stimuli and that there would be a strong correlation between the number of errors a figure incurred in Experiment 1 and the response time measure. (Since both this and the first experiment use verbal presentation, the correlation between these two is a better test of the response-bias hypothesis than is a correlation between Experiment 2 and this one.) The instructions were expected to lower the number of errors, perhaps eliminating the differences in accuracy among the stimuli.

Clearly, there is no problem of response bias in the latency measure. Although subjects still recall the 
stimuli (partly to ensure that they attend to the descriptions), the measure of elapsed time to hear descriptions requires no output by the subject, and so a bias to simplify drawings cannot operate here.

\section{Method}

The method was identical to that of Experiment 1, with the following exceptions. First, the subjects controlled the rate of presentation by saying "OK" when they were ready to hear the element of the next cell. Second, they were told to have a clear mental picture of what had already been described before giving the signal for the next cell. During the practice trials, subjects were questioned to ensure that they followed these instructions. Third, the experimenter started a stopwatch upon saying the element of the first cell and stopped it upon saying the element of the last cell. This time served as the main dependent measure and is called "image construction time."

Ten Stanford undergraduates fulfilled a course requirement by participating in this experiment.

\section{Results}

The elapsed times to hear each description are shown in Table 3. As can be seen, there were again predictable differences between the stimuli: The times ranged from $49 \mathrm{sec}$ to $89 \mathrm{sec}$. The correlation between the image construction time and the number of errors (from Experiment 1) for each figure was $.90[\mathrm{t}(14)=7.73$, $\mathrm{p}<.001]$.

The error analysis, shown in Table 4, revealed few

Table 3

Construction Times in Seconds for Experiment 3: Verbal Descriptions

\begin{tabular}{cccccc}
\hline & \multicolumn{4}{c}{ Number of Edges } \\
\cline { 2 - 3 } \cline { 5 - 6 } & \multicolumn{2}{c}{ Four Parts } & & \multicolumn{2}{c}{ Six Parts } \\
\cline { 2 - 3 } \cline { 5 - 6 } & Under 14 & 14 & & 14 & Over 14 \\
\hline One Figure & & & & \\
Asymmetric & 60.8 & 56.0 & & 77.1 & 76.8 \\
Symmetric & 52.6 & 74.7 & 60.4 & 58.9 \\
Two Figures & & & & & \\
Asymmetric & 48.7 & 58.3 & 68.1 & 88.7 \\
Symmetric & 58.4 & 54.5 & 59.7 & 59.4 \\
Mean & 55.1 & 60.9 & 66.3 & 71.0 \\
\hline
\end{tabular}

Table 4

Errors per Stimulus for Experiment 3: Verbal Descriptions

\begin{tabular}{|c|c|c|c|c|}
\hline & \multicolumn{4}{|c|}{ Number of Edges } \\
\hline & \multicolumn{2}{|c|}{ Four Parts } & \multicolumn{2}{|c|}{ Six Parts } \\
\hline & Under 14 & 14 & 14 & Over 14 \\
\hline \multicolumn{5}{|l|}{ One Figure } \\
\hline Asymmetric & 3.2 & 1.8 & 3.5 & 3.0 \\
\hline Symmetric & 1.0 & 1.8 & 2.6 & 2.3 \\
\hline \multicolumn{5}{|l|}{ Two Figures } \\
\hline Asymmetric & .4 & 3.4 & 3.2 & 4.8 \\
\hline Symmetric & 2.1 & 4.4 & 2.7 & 2.3 \\
\hline Mean & 1.68 & 2.85 & 3.00 & 3.10 \\
\hline
\end{tabular}

differences among the stimuli. An analysis of variance showed that single-figure stimuli incurred fewer errors than those with two figures $(2.4$ vs. 2.9 errors, respectively) $[\mathrm{F}(1,9)=5.71, \mathrm{MSe}=1.84, \mathrm{p}<.05]$ and that the complexity factor was again reliable $[F(3,27)=3.11$, $\mathrm{MSe}=5.64, \mathrm{p}<.05]$. For the four levels of complexity, there were $1.68,2.85,3.00$, and 3.10 errors/stimulus. None of the other effects approached significance (all ps > 10). The correlation between errors in this task and errors in Experiment 1 was .61.

\section{Discussion}

With self-regulation of presentation, subjects may have been able to equalize the recall differences between the figures (to some extent) by spending more time constructing and rehearsing the difficult ones (i.e., a sort of speed-accuracy tradeoff that eliminated the effects of errors).

This experiment strongly implies that the differences between stimuli are the result of encoding or storage processes, rather than occurring at the output stage. The construction time measure is not open to the response-bias explanation, so its high correlation with performance in Experiment 1 gives more evidence for the use of a visual representation.

\section{GENERAL DISCUSSION}

The experiments reported confirm the major hypothesis: When the verbal content of a stimulus is held constant, the visual complexity of the figure still has a marked effect on memory. Does this imply that subjects constructed a quasipictorial representation to remember the figures? It is difficult to imagine why performance should be closely related to visual complexity (most notably, symmetry, edges, and parts) unless some stage of processing involves the use of pictorial information. The alternative hypothesis, that subjects use a verbal grouping strategy to chunk the figure, seems implausible in view of the results of Experiment 2. The close correspondence between results of description and visual presentation leads to the conclusion that the underlying memory representation is similar for the two tasks.

Of course, it might be argued that perceptual analysis results in a propositional representation (e.g., Palmer, 1977), so that the correspondence between Experiments 1 and 2 gives no support to the use of a specifically visual representation. This is not the place to discuss the "analogue-propositional" debate (see Anderson, 1978), but we will point out that such an explanation differs little from our own proposal. If an image is considered to be the same sort of representation as results from higher level perceptual processing, as Kosslyn and Pomerantz (1977) and Shepard (1981) have suggested, then it may make no difference whether one's theory uses a picture metaphor or a "proposition metaphor" to represent it. The important distinction is 
between a representation that embodies the perceptual structure of the stimuli rather than the semantic or informational content alone (see Shepard, 1981, for a full discussion of this issue).

For example, in their present forms, the general amodal theories of memory like HAM (Anderson \& Bower, 1973) and ACT (Anderson, 1976) seem unable to derive perceptual descriptions from input of the basic elements used in our task. Perhaps production systems could be written in ACT to combine the elements to form larger chunks: For example, a lower right next to a lower left element makes a triangle, as do a lower right above an upper right and an upper right next to an upper left, and so on. However, it seems most unlikely that people possess such knowledge in that form (using our terminology for describing the elements), and it is not clear that subjects have a more general form of that knowledge (e.g., that placing congruent right triangles together in certain ways results in larger right triangles pointing in different directions).

A more plausible account seems to be that subjects can imagine two adjacent elements and inspect their image for the resulting unit, if any. Thus, performance is directed by the same principles that direct the parsing and storage of information acquired during perception. For example, readers may introspect on whether they know what results from placing together two congruent trapezoids so that their long sides are adjacent, without actually imagining the figures. As shown in Figure 1-D, the result is a hexagon. That subjects made an average of .5 errors when recalling this stimulus suggests that they used perceptual information to form easily remembered units like "hexagons," since most people appear not to know the abstract rules of the combination of geometric figures.

Pylyshyn (1981) has proposed that subjects' tacit knowledge about spatial relations may explain imagery phenomena in tasks such as mental rotation and imagery scanning. We have argued that people do not have the tacit knowledge to produce the results of our experiments-rather, that these results (as well as those of Podgorny \& Shepard, 1978) arise from emergent properties of the memory representation. Furthermore, the tacit knowledge argument runs into trouble when applied to memory studies: It must claim that subjects "forget" figures they believe to be difficult to remember, so that performance differences reflect subjects' beliefs rather than actual memory processes. However, it seems very unlikely that people willingly perform worse in a task in order to be consistent with their implicit theories, and making recall errors is more obviously poor performance than is creating reaction time differences. Our own subjects seemed to be quite dissatisfied with imperfect performance in the task, often apologizing for their errors. (Also, the social factors in group experiments with immediate feedback favor maximizing performance.) These considerations may not be lethal to the tacit-knowledge argument as applied to memory performance, but the burden of proof appears to rest with its proponents.

Our hypothesis can make no special claim about the form of memory for the "chunks," once they have been formed. It might be that subjects use perceptual representations to deduce structural descriptions of the stimuli, which are then remembered as verbal chunks, like "triangle" and "diagonal bar." Our suspicion is that subjects do not do this, based on their introspections, as well as on the observation that people do not have ready labels for the shapes of the chunks. Simple labels like "trapezoid" or "jagged edges" will not be sufficient to represent a chunk, since they do not give enough information about size or orientation of the part. Longer, more complete labels will be harder to retrieve, thereby mitigating the value of chunking. Thus, although our data do not exclude the possibility of higher level verbal coding, it is difficult to specify verbal codes that would effectively represent the chunks without being as difficult to remember as the original elements.

The second main question of interest was the difference between verbal and visual presentations. Overall there was great similarity, but, not surprisingly, subjects who saw the whole figure were better able to use gestalt features than were those who heard piecemeal descriptions. This was manifested in the greater effect of symmetry in visual presentation and in the greater number of triangle inversions for verbal presentation (in spite of fewer overall errors).

The latter result, in particular, suggests that subjects chunk the elements into parts to reduce the memory load, but that they do this less often with visual presentation. This difference may be due to the small exposure time $(4 \mathrm{sec})$ in the visual presentation condition, but it probably also reflects a greater appreciation of the configural properties of the stimuli. An interesting question for future investigation is the extent to which people are able to derive configural properties from verbal descriptions.

In conclusion, it appears that memory for simple forms involves visual representations, even when the forms are described. When a form is perceptually complex, it is difficult to remember, even when it is constructed from the same elements as less complex forms are; this effect of complexity is remarkably similar for both visual and verbal modes of presentation. The differences between described and perceived stimuli suggest that some global information that is readily perceived is not always computed from a description. Fortunately, English contains words that directly communicate global properties of objects, so that people do not have to deduce those properties from descriptions of parts of objects. This flexibility of language allows easier communication, but it also necessitates the use of unfamiliar forms and strict controls on the verbal content of descriptions in experiments of this 
sort. With these restrictions, we were able to avoid some of the pitfalls in investigating representations in memory for forms.

\section{REFERENCES}

Anderson, J. R. Language, memory and thought. Hillsdale, N.J: Erlbaum, 1976.

ANDERSON, J. R. Arguments concerning representations for mental imagery. Psychological Review, 1978, 85, 249-277.

ANDERson, J. R., \& Bower, G. H. Human associative memory. Washington, D.C: Winston, 1973.

Atrinson, R. C., \& Shiffrin, R. M. Human memory: A proposed system and its control processes. In K. W. Spence \& J. T. Spence (Eds.), Advances in the psychology of learning and motivation (Vol. 2). New York: Academic Press, 1968.

BowER, G. H. Imagery as a relational organizer in associative learning. Journal of Verbal Learning and Verbal Behavior, 1970, 9, 529-533. (a)

Bower, G. H. Mental imagery and associative learning. In L. Gregg (Ed.), Cognition in learning and memory. New York: Wiley, 1970. (b)

Cooper, L. A., \& Shepard, R. N. Chronometric studies of the rotation of mental images. In W. G. Chase (Ed.), Visual information processing. New York: Academic Press, 1973.

Finke, R. A. The functional equivalence of mental images and errors of movement. Cognitive Psychology, 1979, 11, 235-264.

Finke, R. A. Levels of equivalence in imagery and perception. Psychological Review, 1980, 87, 113-132.

Foos, P. W. Constructing cognitive maps from sentences. Journal of Experimental Psychology: Human Learning and Memory, $1980,6,25-38$.

Glanzer, M., \& Cunitz, A. R. Two storage mechanisms in free recall. Journal of Verbal Learning and Verbal Behavior, 1966, 5, 351-360.

Hochberg, J., \& Brooks, V. The psychophysics of form: Reversible-perspective drawings of spatial objects. American Journal of Psychology, 1960, 73, 337-354.
Kosslyn, S. M. Information representation in visual images, Cognitive Psychology, 1975, 7, 341-370.

Kosslyn, S. M., Balt, T. M., \& Reiser, B. J. Visual images preserve metric spatial information: Evidence from studies of imagery scanning. Journal of Experimental Psychology: Human Perception and Performance, 1978, 4, 47-60.

Kosslyn, S. M., \& Pomerantz, J. R. Imagery, propositions, and the form of internal representations. Cognitive Psychology, $1977,9,52-76$.

Nersser, U. Cognition and reality. San Francisco: Freeman, 1976.

Paivio, A. Mental imagery in associative learning and memory. Psychological Review, 1969, 76, 241-263.

Paivio, A. Imagery and verbal processes. New York: Holt, Rinehart \& Winston, 1971.

Palmer, S. E. Hierarchical structure in perceptual representation. Cognitive Psychology, 1977, 9, 441-474.

Podgorny, P., \& ShePaRd, R. N. Functional representations common to visual perception and imagination. Journal of Experimental Psychology: Human Perception and Performance, 1978, 4, 21-35.

Posneh, M. I., Boies, S. J., Eichelman, W. H., \& Taylor, R. I. Retention of visual and name codes of single letters. Journal of Experimental Psychology Monograph, 1969, 79(1, Pt. 2).

Pylyshyn, Z. W. The imagery debate: Analogue media versus tacit knowledge. Psychological Review, 1981, 88, 16-45.

RILEY, D. Memory for form. In L. Postman (Ed.), Psychology in the making. New York: Knopf, 1962.

ShePARd, R. N. Psychophysical complementarity. In M. Kubovy \& J. R. Pomerantz (Eds.), Perceptual organization. Hillsdale, N.J: Erlbaum, 1981.

Stevens, A., \& Coupe, P. Distortions in judged spatial relations. Cognitive Psychology, 1978, 10, 422-437.

TVErSKY, B. Pictorial and verbal encoding in a short-term memory task. Perception \& Psychophysics, 1969, 6, 225-233.

TVERSKY, B. Breadth of pictorial and verbal codes in memory. Bulletin of the Psychonomic Society, 1974, 4, 65-68.

(Received for publication May 1, 1981; revision accepted October 20, 1981.) 